\title{
Weibull Model for Dynamic Pricing in e-Business
}

\author{
Nicholas Nechval ${ }^{1}$, Maris Purgailis ${ }^{1}$, and Konstantin Nechval ${ }^{2}$ \\ ${ }^{1}$ University of Latvia, EVF Research Institute, Statistics Department, \\ Raina Blvd 19, LV-1050 Riga, Latvia \\ nechval@junik. Iv \\ ${ }^{2}$ Transport and Telecommunication Institute, Applied Mathematics Department, \\ Lomonosov Street 1, LV-1019 Riga, Latvia \\ konstan@tsi.lv
}

\begin{abstract}
As is the case with traditional markets, the sellers on the Internet do not usually know the demand functions of their customers. However, in such a digital environment, a seller can experiment different prices in order to maximize his profits. In this paper, we develop a dynamic pricing model to solve the pricing problem of a Web-store, where seller sets a fixed price and buyer either accepts or doesn't buy. Frequent price changes occur due to current market conditions. The model is based on the two-parameter Weibull distribution (indexed by scale and shape parameters), which is used as the underlying distribution of a random variable $X$ representing the amount of revenue received in the specified time period, say, day. In determining (via testing the expected value of $X$ ) whether or not the new product selling price $c$ is accepted, one wants the most effective sample size $n$ of observations $X_{1}, \ldots$, $X_{n}$ of the random variable $X$ and the test plan for the specified seller risk of Type I (probability of rejecting $c$ which is adequate for the real business situation) and seller risk of Type II (probability of accepting $c$ which is not adequate for the real business situation). Let $\mu_{1}$ be the expected value of $X$ in order to accept $c$, and $\mu_{2}$ be the expected value of $X$ in order to reject $c$, where $\mu_{1}>\mu_{2}$, then the test plan has to satisfy the following constraints: (i) $\operatorname{Pr}\left\{\right.$ statistically reject $\left.c \mid E\{X\}=\mu_{1}\right\}=\alpha_{1}$ (seller risk of Type I), and (ii) $\operatorname{Pr}\left\{\right.$ statistically accept $\left.c \mid E\{X\}=\mu_{2}\right\}=\alpha_{2}$ (seller risk of Type II). It is assumed that $\alpha_{1}<0.5$ and $\alpha_{2}<0.5$. The cases of product pricing are considered when the shape parameter of the two-parameter Weibull distribution is assumed to be a priori known as well as when it is unknown.
\end{abstract}

Keywords: e-business, pricing, uncertainty, revenue, Weibull model, seller risk, test plan.

\section{Introduction}

The benefits of dynamic pricing are twofold. First, it provides new opportunities for companies to maximize their return per customer. With lower menu costs (that is, the cost of displaying prices to customers), companies can have multiple prices for different channels and product configurations - and can change those prices more 
frequently. Companies able to gather information about their competition and about customer needs and willingness to pay can customize their offerings and prices. This enables them to deploy dynamic pricing through the most appropriate of many channels. With dynamic pricing, companies can give their customers exactly what they want, at exactly the price they are willing to bear. Nothing is left on the table. The second, perhaps less obvious, benefit is that dynamic pricing can also bring better returns on deployed assets. For businesses with high fixed-cost technology infrastructures, periods of low demand and, thus, low utilization are expensive. Conversely, when there are inflexibilities in the supply chain for critical components, periods of high demand can lead to shortages and can both delay purchasing and damage customer relationships. But with dynamic pricing, companies can encourage demand in slow periods and discourage it in busy periods.

Pricing in e-business is similar to that in traditional business. However, in ebusiness prices are readily available to consumers and competing organizations via the Internet. With vital pricing data readily available, organizations may be forced to reevaluate their pricing methodology and strategy to avoid being significantly undercut, while remaining competitive in their respective markets.

On Internet markets, as is the case on most traditional markets, sellers do not usually know the demand functions of their customers without ambiguity. So as to establish a reasonable theory of the way Web-stores should fix their prices on the network, it is thus necessary to take the uncertainty they face into account by explicitly introducing the process by which they acquire information on their environment. In most uncertain environments, Web-stores can only learn what the most profitable prices are by price experimentations. By its very nature, the Internet is well adapted to such a learning process. Indeed, in this electronic environment the menu costs are negligible and sellers can therefore easily experiment different prices. However, as such a learning activity is costly, the Web-stores have to make a tradeoff between charging the most profitable price according to their current information (i.e., to exploit their information) and inquiring on the profitability of the other prices (i.e., to explore the profitability of the other prices). The determination of the Web-stores' pricing strategy under uncertainty is thus an inter-temporal decision problem in which they have to make an exploration vs. exploitation compromise. Some dynamic pricing algorithms have already been proposed in the E-commerce literature.

Much recent work in dynamic pricing has concentrated on pricing perishable products, such as are typically sold by the airline, hotel, car rental, and fashion industries. Inventory levels are a critical consideration in pricing such products. A large literature has addressed dynamic pricing in the presence of inventory considerations, where it is usually referred to as "revenue management" or "yield management." A recent comprehensive review of such practices is given in [1]; also see [2].

In a classic paper, Rothschild [3] investigated the nature of optimal sequential pricing strategies that experiment with two prices. He showed that under a general class of prior distributions on demand, there is a positive probability that a pricing policy that maximizes expected total discounted reward over an infinite horizon will select the revenue-maximizing price only finitely many times. Therefore, the policy may converge to the suboptimal price. This phenomenon was studied more generally by Easley and Kiefer [4, 5], as well as McLennan [6] and Aghion et al. [7]. Several 
other prior studies have also described the qualitative behavior of optimal priceadjustment strategies using parametric classes of demand functions, such as those by Grossman, Kihlstrom, and Mirman [8], Mirman, Samuelson, and Urbano [9]. Leloup and Deveaux [10] consider pricing strategies in a Bayesian framework.

In this paper, however, we shall consider markets where inventories are not as important to pricing and focus on the revenue implications of demand functions online. By taking a frequentist approach, our methods contrast with some line search-type methods previously proposed for this problem in the literature, such as the "derivative following" methods proposed by Greenwald and Kephart [11], Dasgupta and Das [12]. These methods resemble stochastic approximation algorithms for locating the revenuemaximizing price. While simple to implement, these methods do not incorporate prior information, nor do they make full use of the data that have been observed.

\section{Model Formulation}

Consider a seller (vendor) operating a shop where a single product is for sale. Here, a shop is understood to be any space, either real or virtual, where prices are displayed to arriving customers, who then decide whether to purchase the product. For example, a shop may be a web page or collection of web pages that display the price for a particular product, such as a software product or digital media document, and provides visitors with links to purchase the product. We assume that information about present and past prices is not available outside of the shop, as would be good practice if the seller wishes to strictly control the price of the product. In each of consecutive time periods of equal length (say, day), a random number of customers arrive to the shop, observe the posted price, and individually decide to either purchase some quantity of the product or exit the shop without purchasing. We shall further assume that the numbers of customers arriving in each period form an identically distributed sequence that is independent of past arrivals as well as both past and current prices. This assumption may be warranted in a finite time horizon if we assume that the customer base is composed, for example, of a large number of distributed Internet users, each of whom desires the good in a given period with a small probability, independent of other users and independent of past demand. We also assume that the number of consecutive time periods of equal length represents a deterministic time horizon (which may be infinite if no definite end period is anticipated). The seller may change the price at the start of any time period, always choosing from a predetermined setof prices. Given that the price is only displayed in the shop, we assume there is no cost to changing the price. A customer will buy the product if the current price is less than or equal to his or her privately held reservation price. We shall assume that the reservation prices of arriving customers also form an identically distributed sequence that is independent of past prices, the reservation prices of past customers, and past numbers of customer arrivals.

In some time period, which consists of $n$ consecutive time periods of equal length, the seller sets a new price $c$ for product and receives a random amount of revenue according to the number of customers arriving to the shop in that period whose 
reservation prices are at least as great as $c$. The seller can only observe a random amount of revenue, $X$, received in each of $n$ consecutive time periods of equal length. Thus, he will have available a sample of observations $\mathrm{X}=\left(X_{1}, \ldots, X_{n}\right)$. It is assumed that the random variable $X$ follows the two-parameter Weibull distribution with the probability density function

$$
f(x \mid \beta, \delta)=\frac{\delta}{\beta}\left(\frac{x}{\beta}\right)^{\delta-1} \exp \left[-\left(\frac{x}{\beta}\right)^{\delta}\right](x>0)
$$

indexed by scale and shape parameters $\beta$ and $\delta$.

The problem is to find the sample size $n$ of observations of the random variable $X$ and test plan based on $\mathrm{X}=\left(X_{1}, \ldots, X_{n}\right)$ in order to determine whether or not the new product selling price $c$ is accepted. The test plan has to satisfy the specified constraints for the seller risks of Type I and II, respectively.

\section{Test Plan When the Shape Parameter Is a Priori Known}

In determining whether or not the new product selling price $c$ is accepted, we have to use the test plan which satisfies the following conditions:

$$
\begin{aligned}
& \operatorname{Pr}\left\{\text { statistically reject } c \mid E\{X\}=\mu_{1}\right\}=\alpha_{1} \quad \text { (seller risk of Type I), } \\
& \operatorname{Pr}\left\{\text { statistically accept } c \mid E\{X\}=\mu_{2}\right\}=\alpha_{2} \text { (seller risk of Type II), }
\end{aligned}
$$

where

$$
E\{X\}=\mu=\int_{0}^{\infty} x f(x \mid \beta, \delta) d x=\beta \Gamma\left(1+\frac{1}{\delta}\right)
$$

is the expected value of $X, \quad E\{X\}=\mu_{1}$ is statistically acceptable for $c, E\{X\}=\mu_{2}$ is statistically rejectable for $c, \alpha_{1}$ and $\alpha_{2}$ are the specified seller risks of Types I and II, respectively.

To test for assuring $\mu$ in the Weibull distribution, let $n$ observations of the random variable $X$ be made and the complete data sample $\mathrm{X}=\left(X_{1}, X_{2}, \ldots, X_{n}\right)$ be obtained. Then, if the shape parameter $\delta$ is known a priori, the maximum likelihood estimator of $\beta^{\delta}$ is given by

$$
\widehat{\beta}^{\delta}=\sum_{i=1}^{n} X_{i}^{\delta} / n
$$

and the pivotal quantity

$$
V=2 n\left(\widehat{\beta}^{\delta} / \beta^{\delta}\right)
$$

is $\chi^{2}$ distributed with $2 n$ degrees of freedom. The $n$ and $h$ are determined by 


$$
\left\{\begin{array}{l}
\widehat{\beta}^{\delta}>h, \quad \text { accept } c, \\
\widehat{\beta}^{\delta} \leq h, \quad \text { reject } c .
\end{array}\right.
$$

To select $n$ and $h$, we use the equations (2) and (3), which can be presented in the case when the parameter $\delta$ is known as

$$
\begin{gathered}
\operatorname{Pr}\left\{\widehat{\beta}^{\delta} \leq h \mid \mu=\mu_{1} ; \delta\right\}=\operatorname{Pr}\left\{\frac{2 n \widehat{\beta}^{\delta}}{\beta_{1}^{\delta}} \leq \frac{2 n h}{\beta_{1}^{\delta}} \mid \mu=\mu_{1} ; \delta\right\}=\operatorname{Pr}\left\{V \leq \chi_{2 n ; \alpha_{1}}^{2}\right\}=\alpha_{1}, \\
\operatorname{Pr}\left\{\widehat{\beta}^{\delta}>h \mid \mu=\mu_{2} ; \delta\right\}=\operatorname{Pr}\left\{\frac{2 n \widehat{\beta}^{\delta}}{\beta_{2}^{\delta}}>\frac{2 n h}{\beta_{2}^{\delta}} \mid \mu=\mu_{2} ; \delta\right\}=\operatorname{Pr}\left\{V>\chi_{2 n ; 1-\alpha_{2}}^{2}\right\}=\alpha_{2},
\end{gathered}
$$

where

$$
\beta_{j}=\frac{\mu_{j}}{\Gamma(1+1 / \delta)}, \quad j=1,2,
$$

$\chi_{2 n ; p}^{2}$ is the $p$-quantile of $\chi^{2}$ distribution with $2 n$ degrees of freedom. From (8) and (9),

$$
\frac{\beta_{2}^{\delta}}{\beta_{1}^{\delta}} \leq \frac{\chi_{2 n ; \alpha_{1}}^{2}}{\chi_{2 n ; 1-\alpha_{2}}^{2}}
$$

Therefore, the required $n$ is the smallest integer satisfying (11). Using $n$ based on (11), we have from (8) an (10) that the $h$ for $\widehat{\beta}^{\delta}$ is given by

$$
h=\left(\frac{\mu_{1}}{\Gamma(1+1 / \delta)}\right)^{\delta} \frac{\chi_{2 n ; \alpha_{1}}^{2}}{2 n} .
$$

Thus, the new product selling price $c$ is accepted if $\widehat{\beta}^{\delta}>h$; otherwise, it is rejected.

\section{Test Plan When the Shape Parameter Is Unknown}

\subsection{Preliminaries}

We consider both parameters $\beta, \delta$ to be unknown. We will give here appropriate expressions for the case of complete (uncensored) sampling. Let $\mathbf{X}=\left(X_{1}, \ldots, X_{n}\right)$ be a random sample from the two-parameter Weibull distribution (1), and let $\widehat{\beta}, \widehat{\delta}$ be maximum likelihood estimates of $\beta, \delta$ computed on the basis of $\mathbf{X}=\left(X_{1}, \ldots, X_{n}\right)$. In terms of the Weibull variates, we have that

$$
V_{1}=(\widehat{\beta} / \beta)^{\delta}, \quad V_{2}=\delta / \widehat{\delta}, \quad V_{3}=(\widehat{\beta} / \beta)^{\widehat{\delta}}
$$

are pivotal quantities. Furthermore, let

$$
Z_{i}=\left(X_{i} / \widehat{\beta}\right)^{\hat{\delta}}, \quad i=1, \ldots, n .
$$


It is readily verified that any $n-2$ of the $Z_{i}^{\prime}$ 's, say $Z_{i}, \ldots, Z_{n-2}$ form a set of $n-2$ functionally independent ancillary statistics. The appropriate conditional approach, first suggested by Fisher [13], is to consider the distributions of $V_{1}, V_{2}, V_{3}$ conditional on the observed value of $\mathbf{Z}=\left(Z_{i}, \ldots, Z_{n}\right)$. (For purposes of symmetry of notation we include all of $Z_{i}, \ldots, Z_{n}$ in expressions stated here; it can be shown that $Z_{n}, Z_{n-1}$, can be determined as functions of $Z_{i}, \ldots, Z_{n-2}$ only.)

The marginal distribution of

$$
V_{2}=\delta / \hat{\delta}
$$

conditional on fixed $\mathbf{z}=\left(z_{i}, \ldots, z_{n}\right)$ is appropriate for making inference about $\delta$ when $\beta$ is unknown. It can be found (see Corollary 1.1 below with $r=n$ ) that the marginal probability density function of $V_{2}$, conditional on fixed $\mathbf{z}$, is given by

$$
g_{2}\left(v_{2} \mid \mathbf{z}\right)=\vartheta(\mathbf{z}) v_{2}^{n-2} \prod_{i=1}^{n} z_{i}^{v_{2}}\left(\sum_{i=1}^{n} z_{i}^{v_{2}}\right)^{-n}, \quad\left(v_{2}>0\right),
$$

where

$$
\vartheta(\mathbf{z})=\left[\int_{0}^{\infty} v_{2}^{n-2} \prod_{i=1}^{n} z_{i}^{v_{2}}\left(\sum_{i=1}^{n} z_{i}^{v_{2}}\right)^{-n} d v_{2}\right]^{-1}
$$

is the normalizing constant. The probability statement

$$
\operatorname{Pr}\left\{q_{1} \leq V_{2} \leq q_{2} \mid \mathbf{z}\right\}=1-\alpha
$$

leads in the usual way to the $(1-\alpha)$ conditional confidence interval

$$
q_{1} \widehat{\delta} \leq \delta \leq q_{2} \widehat{\delta}
$$

The marginal distribution of

$$
V_{3}=(\widehat{\beta} / \beta)^{\widehat{\delta}}
$$

is used to make inferences about $\beta$, when $\delta$ is unknown. It can be found (see Corollary 2.1 below with $r=n$ ) that the probability distribution function of $V_{3}$, conditional on fixed $\mathbf{z}$, can conveniently be expressed as

$$
\operatorname{Pr}\left\{V_{3} \leq h \mid \mathbf{z}\right\}=\vartheta(\mathbf{z}) \int_{0}^{\infty} \frac{v_{2}^{n-2}\left(\prod_{i=1}^{n} z_{i}^{v_{2}}\right) G_{n}\left(h^{v_{2}} \sum_{i=1}^{n} z_{i}^{v_{2}}\right)}{\left(\sum_{i=1}^{n} z_{i}^{v_{2}}\right)^{n}} d v_{2} \quad(h \geq 0),
$$

where $G_{n}(\tau)$ represents the incomplete gamma integral,

$$
G_{n}(\tau)=\int_{0}^{\tau} \frac{t^{n-1} e^{-t}}{\Gamma(n)} d t
$$


For any specified value of $h$, (21) can be integrated numerically to give conditional probability statements for $V_{3}$. Then, for example, the probability statement

$$
\operatorname{Pr}\left\{V_{3} \leq h \mid \mathbf{z}\right\}=\alpha
$$

leads to an $\alpha$ conditional confidence interval for $\beta$,

$$
\beta \geq h^{-1 / \hat{\delta}} \hat{\beta} \text {. }
$$

Main Theorems. The results can be presented more naturally, however, if we consider the distribution of the logarithm of a Weibull variate, which we denote by $Y$. The random variate $Y$ follows the first asymptotic distribution of extreme values, with density

$$
f(y \mid a, b)=\frac{1}{b} \exp \left(\frac{y-a}{b}\right) \exp \left(-\exp \left(\frac{y-a}{b}\right)\right)(-\infty<y<\infty),
$$

where

$$
a=\ln \beta, \quad b=\delta^{-1}
$$

Now, (25) is a distribution with location and scale parameters $a$ and $b$, respectively, and it is well known that if $\hat{a}, \hat{b}$ are maximum likelihood estimates for $a, b$, from a complete (uncensored) sample of size $n$, then

$$
U_{1}=(\hat{a}-a) / b, \quad U_{2}=\hat{b} / b, \quad U_{3}=(\hat{a}-a) / \hat{b}
$$

are pivotal quantities, whose distributions depend only on $n$. That is, inferences concerning $a$ and $b$ here may be based on the pivotal quantities (27) as usual; however, the distributions of these pivotals should be considered conditional on the observed values of ancillary statistics. We generalize this situation and allow the original data to be possibly censored. Here the following theorems hold.

Theorem 1. Let $Y_{1} \leq \ldots \leq Y_{r}$ be the first $r$ ordered past observations from a sample of size $n$ from the first asymptotic distribution of extreme values (25). Then the marginal probability density function of the pivotal quantity

$$
U_{2}=\hat{b} / b
$$

conditional on fixed

$$
\mathbf{s}=\left(s_{1}, \ldots, s_{r}\right) \text {, }
$$

where

$$
S_{i}=\left(Y_{i}-\hat{a}\right) / \hat{b}, \quad i=1, \ldots, r,
$$

are ancillary statistics, any $r-2$ of which form a functionally independent set, $\hat{a}$ and $\bar{b}$ are the maximum likelihood estimators of $a$ and $b$ based on the first $r$ ordered past 
observations $\left(Y_{1} \leq \ldots \leq Y_{r}\right)$ from a sample of size $n$ from the first asymptotic distribution of extreme values (25), which can be found from solution of

$$
\widehat{a}=\hat{b} \ln \left(\left[\sum_{i=1}^{r} e^{y_{i} / \hat{b}}+(n-r) e^{y_{r} / \hat{b}}\right] / r\right)
$$

and

$$
\hat{b}=\left(\sum_{i=1}^{r} y_{i} e^{y_{i} / \hat{b}}+(n-r) y_{r} e^{y_{r} / \hat{b}}\right)\left(\sum_{i=1}^{r} e^{y_{i} / \hat{b}}+(n-r) e^{y_{r} / \hat{b}}\right)^{-1}-\frac{1}{r} \sum_{i=1}^{r} y_{i},
$$

is given by

$$
\begin{gathered}
g_{2}\left(u_{2} \mid \mathbf{s}\right)=\vartheta(\mathbf{s}) u_{2}^{r-2} \exp \left(u_{2} \sum_{i=1}^{r} s_{i}\right)\left[\sum_{i=1}^{r} \exp \left(s_{i} u_{2}\right)+(n-r) \exp \left(s_{r} u_{2}\right)\right]^{-r}, \\
u_{2} \in(0, \infty),
\end{gathered}
$$

where

$$
\vartheta(\mathbf{s})=\left(\int_{0}^{\infty} u_{2}^{r-2} \exp \left(u_{2} \sum_{i=1}^{z} s_{i}\right)\left[\sum_{i=1}^{r} \exp \left(s_{i} u_{2}\right)+(n-r) \exp \left(s_{r} u_{2}\right)\right]^{-r} d u_{2}\right)^{-1}
$$

is the normalizing constant.

Proof. The joint density of $Y_{1} \leq \ldots \leq Y_{r}$ is given by

$$
f\left(y_{1}, \ldots, y_{r} \mid a, b\right)=\frac{n !}{(n-r) !} \prod_{i=1}^{r} \frac{1}{b} \exp \left(\frac{y_{i}-a}{b}-\exp \left(\frac{y_{i}-a}{b}\right)\right) \exp \left(-(n-r) \exp \left(\frac{y_{r}-a}{b}\right)\right)
$$

Using the invariant embedding technique [14-21], we then find in a straightforward manner, that the probability element of the joint density of $U_{1}, U_{2}$, conditional on fixed $\mathbf{s}=\left(s_{1}, \ldots, s_{r}\right)$, is

$$
f\left(u_{1}, u_{2} \mid \mathbf{s}\right) d u_{1} d u_{2}
$$$$
=\vartheta^{\circ}(\mathbf{s}) u_{2}^{r-2} \exp \left(u_{2} \sum_{i=1}^{r} s_{i}\right) e^{r u_{1}} \exp \left(-e^{u_{1}}\left[\sum_{i=1}^{r} \exp \left(s_{i} u_{2}\right)+(n-r) \exp \left(s_{r} u_{2}\right)\right]\right) d u_{1} d u_{2},
$$

where

$$
u_{1} \in(-\infty, \infty), \quad u_{2} \in(0, \infty)
$$

$$
\vartheta^{\circ}(\mathbf{s})=\left(\int_{0}^{\infty} \Gamma(r) u_{2}^{r-2} \exp \left(u_{2} \sum_{i=1}^{z} s_{i}\right)\left[\sum_{i=1}^{r} \exp \left(s_{i} u_{2}\right)+(n-r) \exp \left(s_{r} u_{2}\right)\right]^{-r} d u_{2}\right)^{-1}
$$

is the normalizing constant. Now $u_{1}$ can be integrated out of (36) in a straightforward way to give (33). This ends the proof. 
Corollary 1.1. Let $X_{1} \leq \ldots \leq X_{r}$ be the first $r$ ordered past observations from a sample of size $n$ from the two-parameter Weibull distribution (1). Then the marginal probability density function of the pivotal quantity

$$
V_{2}=\delta / \widehat{\delta}
$$

conditional on fixed

$$
\mathbf{z}=\left(z_{i}, \ldots, z_{n}\right),
$$

where

$$
Z_{i}=\left(X_{i} / \widehat{\beta}\right)^{\hat{\delta}}, \quad i=1, \ldots, r
$$

are ancillary statistics, any $r-2$ of which form a functionally independent set, $\bar{\beta}$ and $\widehat{\delta}$ are the maximum likelihood estimators of $\beta$ and $\delta$ based on the first $r$ ordered past observations $\left(X_{1} \leq \ldots \leq X_{r}\right)$ from a sample of size $n$ from the two-parameter Weibull distribution (1), which can be found from solution of

$$
\widehat{\beta}=\left(\left[\sum_{i=1}^{r} x_{i}^{\widehat{\delta}}+(n-r) x_{r}^{\hat{\delta}}\right] / r\right)^{1 / \hat{\delta}}
$$

and

$$
\widehat{\delta}=\left[\left(\sum_{i=1}^{r} x_{i}^{\hat{\delta}} \ln x_{i}+(n-r) x_{r}^{\widehat{\delta}} \ln x_{r}\right)\left(\sum_{i=1}^{r} x_{i}^{\hat{\delta}}+(n-r) x_{r}^{\hat{\delta}}\right)^{-1}-\frac{1}{r} \sum_{i=1}^{r} \ln x_{i}\right]^{-1},
$$

is given by

$$
g_{2}\left(v_{2} \mid \mathbf{z}\right)=\vartheta(\mathbf{z}) v_{2}^{r-2} \prod_{i=1}^{r} z_{i}^{v_{2}}\left(\sum_{i=1}^{r} z_{i}^{v_{2}}+(n-r) z_{r}^{v_{2}}\right)^{-r}, \quad\left(v_{2}>0\right),
$$

where

$$
\vartheta(\mathbf{z})=\left[\int_{0}^{\infty} v_{2}^{r-2} \prod_{i=1}^{r} z_{i}^{v_{2}}\left(\sum_{i=1}^{r} z_{i}^{v_{2}}+(n-r) z_{z}^{v_{2}}\right)^{-r} d v_{2}\right]^{-1}
$$

is the normalizing constant.

Theorem 2. Let $Y_{1} \leq \ldots \leq Y_{r}$ be the first $r$ ordered past observations from a sample of size $n$ from the first asymptotic distribution of extreme values (25). Then the probability distribution function of the pivotal quantity

$$
U_{3}=(\widehat{a}-a) / \widehat{b},
$$


conditional on fixed $\mathbf{s}$, is given by

$$
=\vartheta(\mathbf{s}) \int_{0}^{\infty} \frac{\operatorname{Pr}\left(U_{3} \leq h \mid \mathbf{s}\right)}{\left[\sum_{i=1}^{r-2} \exp \left(u_{2} \sum_{i=1}^{r} s_{i}\right) G_{r}\left(\exp \left(h u_{2}\right)\left[\sum_{i=1}^{r} \exp \left(s_{i} u_{2}\right)+(n-r) \exp \left(s_{r} u_{2}\right)\right]\right)\right.} d u_{2} \quad(h \geq 0),
$$

where

$$
G_{r}(\tau)=\int_{0}^{\tau} \frac{t^{r-1} e^{-t}}{\Gamma(r)} d t
$$

represents the incomplete gamma integral.

Proof. The proof is similar to that of Theorem 1 and so it is omitted here.

Corollary 2.1. Let $X_{1} \leq \ldots \leq X_{r}$ be the first $r$ ordered past observations from a sample of size $n$ from the from the two-parameter Weibull distribution (1). Then the probability distribution function of the pivotal quantity

$$
V_{3}=(\widehat{\beta} / \beta)^{\widehat{\delta}}
$$

conditional on fixed $\mathbf{z}$, is given by

$$
\operatorname{Pr}\left\{V_{3} \leq h \mid \mathbf{z}\right\}=\vartheta(\mathbf{z}) \int_{0}^{\infty} \frac{v_{2}^{r-2}\left(\prod_{i=1}^{r} z_{i}^{v_{2}}\right) G_{r}\left(h^{v_{2}}\left[\sum_{i=1}^{r} z_{i}^{v_{2}}+(n-r) z_{r}^{v_{2}}\right]\right)}{\left(\sum_{i=1}^{r} z_{i}^{v_{2}}+(n-r) z_{r}^{v_{2}}\right)^{r}} d v_{2} \quad(h \geq 0) .
$$

\subsection{Test Plan}

The $n$ and $h$ are determined by

$$
\left\{\begin{array}{l}
\widehat{\beta}^{\widehat{\delta}}>h, \quad \text { accept } c, \\
\widehat{\beta}^{\widehat{\delta}} \leq h, \quad \text { reject } c .
\end{array}\right.
$$

To select $n$ and $h$, we use the equations (2) and (3), which can be presented in the case when the parameter $\delta$ is unknown as 


$$
\begin{gathered}
\operatorname{Pr}\left\{\widehat{\beta}^{\widehat{\delta}} \leq h \mid \mu=\mu_{1} ; \mathbf{z}\right\}=\operatorname{Pr}\left\{\frac{\widehat{\beta}^{\hat{\delta}}}{\beta_{1}^{\delta}} \leq \frac{h}{\beta_{1}^{\delta}} \mid \mu=\mu_{1} ; \mathbf{z}\right\}=\operatorname{Pr}\left\{V_{3} \leq V_{3\left(\mathbf{z}(n) ; \alpha_{1}\right)}\right\}=\alpha_{1}, \\
\operatorname{Pr}\left\{\widehat{\beta}^{\delta}>h \mid \mu=\mu_{2} ; \mathbf{z}\right\}=\operatorname{Pr}\left\{\frac{\widehat{\beta}^{\delta}}{\beta_{2}^{\delta}}>\frac{h}{\beta_{2}^{\delta}} \mid \mu=\mu_{2} ; \mathbf{z}\right\}=\operatorname{Pr}\left\{V_{3}>V_{3\left(\mathbf{z}(n) ; 1-\alpha_{2}\right)}\right\}=\alpha_{2},
\end{gathered}
$$

where

$$
\operatorname{Pr}\left\{V_{3} \leq V_{3(\mathbf{z}(n) ; \alpha)} \mid \mathbf{z}\right\}=\alpha .
$$

From (51) and (52),

$$
\frac{\beta_{2}^{\widehat{\delta}}}{\beta_{1}^{\widehat{\delta}}} \leq \frac{V_{3\left(\mathbf{z}(n) ; \alpha_{1}\right)}}{V_{3\left(\mathbf{z}(n) ; 1-\alpha_{2}\right)}} .
$$

Therefore, the required $n$ is the smallest integer satisfying (54). Using $n$ based on (54), we have from (51) and (10) that the $h$ for $\widehat{\beta}^{\widehat{\delta}}$ is given by

$$
h=\left(\mu_{1} / E\left\{\Gamma\left(1+\frac{1}{\hat{\delta} V_{2}}\right)\right\}\right)^{\widehat{\delta}} V_{3\left(\mathbf{z}(n) ; \alpha_{1}\right)},
$$

where

$$
E\left\{\Gamma\left(1+\frac{1}{\delta V_{2}}\right)\right\}=\int_{0}^{\infty} \Gamma\left(1+\frac{1}{\delta v_{2}}\right) g_{2}\left(v_{2} \mid \mathbf{z}\right) d v_{2} .
$$

Thus, the new product selling price $c$ is accepted if $\widehat{\beta}^{\hat{\delta}}>h$; otherwise, it is rejected.

\section{Conclusions and Directions for Future Research}

The choice of an appropriate dynamic pricing model is a crucial decision problem because a good model not only can help sellers save testing time and reduce testing cost, but it also can positively affect the image of the product and thus attract more consumers to buy this product. Therefore often the Bayesian approach is used to solve the above problem. Unfortunately, in this case the subjectivity of investigator (a limitation of the Bayesian approach) is introduced through a priori distribution. In order to rule out the subjectivity of investigator and to consider comprehensively the relevant risks, in this paper a frequentist (non-Bayesian) decision analysis is employed. It is based on the invariant embedding technique [14-21] which allows one to use available statistical information as completely as possible.

To extend the scope of our analysis, it would be interesting to explicitly introduce price competition between Web-stores. Indeed, the Internet environment may be a 
very competitive market and the sellers have to take the behaviors of their competitors into account in their own dynamic pricing strategy. So as to take this competition into account in our model, we can introduce a non-stationary behavior of the customers on the market. Such an extension would be a very big step towards a generalization of the pricing model proposed in this paper.

Acknowledgments. This research was supported in part by Grant No. 06.1936, Grant No. 07.2036, Grant No. 09.1014, and Grant No. 09.1544 from the Latvian Council of Science and the National Institute of Mathematics and Informatics of Latvia. The authors are also very grateful to two anonymous referees for their detailed comments that have helped in improving an earlier version of this paper.

\section{References}

1. Elmaghraby, W., Keskinocak, P.: Dynamic Pricing in the Presence of Inventory Considerations: Research Overview, Current Practices and Future Directions. Manage. Sci. 49, 1287-1309 (2003)

2. McGill, J.I., van Ryzin, G.J.: Revenue Management: Research Overview and Prospects. Transport Sci. 33, 233-256 (1999)

3. Rothschild, M.: A Two-Armed Bandit Theory of Market Pricing. J. Econ. Theo. 9, 185202 (1974)

4. Easley, D., Kiefer, N.M.: Controlling a Stochastic Process with Unknown Parameters. Econometrica 56, 1045-1064 (1988)

5. Easley, D., Kiefer, N.M.: Optimal Learning with Endogenous Data. Int. Econ. Rev. 30, 963-978 (1989)

6. McLennan, A.: Price Dispersion and Incomplete Learning in the Long Run. J. Econ. Dynam. Contr. 7, 331-347 (1984)

7. Aghion, P., et al.: Optimal Learning by Experimentation. Rev. Econ. Stud. 58, 621-654 (1991)

8. Grossman, S.J., Kihlstrom, R.E., Mirman, L.J.: A Bayesian Approach to the Production of Information and Learning by Doing. Rev. Econ. Stud. 44, 533-547 (1977)

9. Mirman, L.J., Samuelson, L., Urbano, A.: Monopoly Experimentation. Int. Econ. Rev. 34, 549-563 (1993)

10. Leloup, B., Deveaux, L.: Dynamic Pricing on the Internet: Theory and Simulation. J. Electron. Comm. Res. 1, 53-64 (2001)

11. Greenwald, A.R., Kephart, J.O.: Shopbots and Pricebots. In: Proceedings of the 5th International Conference on Artificial Intelligence (IJCAI 1999), pp. 506-511. Morgan Kaufmann, San Mateo (1999)

12. Dasgupta, P., Das, R.: Dynamic Pricing with Limited Competitor Information in a Multiagent Economy. In: 4th International Conference on Coordination Languages and Models (COORDINATION 2000). Springer, New York (2000)

13. Fisher, R.A.: Two New Properties of Mathematical Likelihood. Proceedings of the Royal Society A 144, 285-307 (1934)

14. Nechval, N.A., Nechval, K.N., Vasermanis, E.K.: Effective State Estimation of Stochastic Systems. Kybernetes 32, 666-678 (2003)

15. Nechval, N.A., Vasermanis, E.K.: Improved Decisions in Statistics. SIA, Izglitibas (2004) 
16. Nechval, N.A., Nechval, K.N.: Constrained Optimization in Newsboy Problems under Uncertainty via Statistical Inference Equivalence Principle. In: Al-Begain, K., Bolch, G., Telek, M. (eds.) Proceedings of the 12th International Conference on Analytical and Stochastic Modelling Techniques and Applications (ASMTA 2005), pp. 166-171. RTU, Riga (2005)

17. Nechval, N.A., Berzins, G., Purgailis, M., Nechval, K.N.: Improved Estimation of State of Stochastic Systems via Invariant Embedding Technique. WSEAS Transactions on Mathematics 7, 141-159 (2008)

18. Nechval, N.A., Berzins, G., Purgailis, M., Nechval, K.N., Zolova, N.: Improved Adaptive Control of Stochastic Systems. Advances in Systems Science and Applications 9, 11-20 (2009)

19. Nechval, N.A., Purgailis, M., Berzins, G., Cikste, K., Krasts, J., Nechval, K.N.: Invariant Embedding Technique and Its Applications for Improvement or Optimization of Statistical Decisions. In: Al-Begain, K., Fiems, D., Knottenbelt, W.J. (eds.) ASMTA 2010. LNCS, vol. 6148, pp. 306-320. Springer, Heidelberg (2010)

20. Nechval, N.A., Purgailis, M., Cikste, K., Berzins, G., Nechval, K.N.: Optimization of Statistical Decisions via an Invariant Embedding Technique. In: Proceedings of the World Congress on Engineering 2010. Lecture Notes in Engineering and Computer Science, London, U.K, pp. 1776-1782 (2010)

21. Nechval, N.A., Nechval, K.N., Purgailis, M., Rozevskis, U.: Improvement of Inventory Control under Parametric Uncertainty and Constraints. In: Dobnikar, A., Lotrič, U., Šter, B. (eds.) ICANNGA 2011, Part II. LNCS, vol. 6594, pp. 136-146. Springer, Heidelberg (2011) 